\title{
Changes in Mucin Production in Human Airway Epithelial Cells After Exposure to Electronic Cigarette Vapor With or Without Nicotine
}

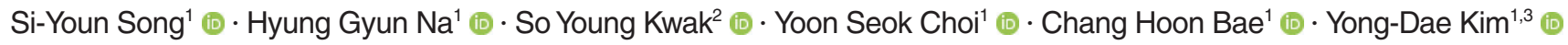 \\ Departments of ${ }^{1}$ Otorhinolaryngology-Head and Neck Surgery and ${ }^{2}$ Medical Science, Yeungnam University College of Medicine, Daegu; \\ ${ }^{3}$ Regional Center for Respiratory Diseases, Yeungnam University Medical Center, Daegu, Korea
}

Objectives. The emergence of electronic cigarettes (e-cigarettes) has created new perceptions of the tobacco market. Unlike traditional tobacco, the greatest advantage of e-cigarettes is that they have less smell and are convenient and inexpensive. Most e-cigarette smokers believe that e-cigarette smoking is less harmful than traditional smoking. Information on the effects of e-cigarettes on human health is limited, and the issue remains controversial.

Methods. We studied the effects of e-cigarette vapor on mucin (MUC5AC and MUC5B) and the change of MUC5AC and MUC5B from e-cigarette liquid with or without nicotine in respiratory epithelial cells. The effects of e-cigarette vapor with or without nicotine on mucin, along with the involved signaling pathways, were investigated using reverse transcriptase-polymerase chain reaction (PCR), real-time PCR, enzyme immunoassays, and immunoblot analysis with several specific inhibitors and small interfering RNA.

Results. E-cigarette vapor with or without nicotine stimulated MUC5AC, but not MUC5B, expression in respiratory epithelial cells. In addition, we showed that e-cigarette vapor with and without nicotine induced MUC5AC expression via activation of the mitogen-activated protein kinase (MAPK; extracellular signal-regulated kinase [ERK] 1/2 and p38) and nuclear factor kappa-light-chain-enhancer of activated B cells (NF-kB) signaling pathways in human airway epithelial cells.

Conclusion. E-cigarette vapor with and with nicotine significantly increased MUC5AC expression in human airway epithelial cells.

Keywords. E-Cigarette Vapor; MUC5AC; Mitogen-Activated Protein Kinase; NF-Kappa B; Epithelial Cell

\section{INTRODUCTION}

Nicotine, a popular addictive constituent of tobacco, is a naturally produced plant alkaloid that comprises approximately $0.6 \%-3.0 \%$ of tobacco. Nicotine was initially used as an insecticide; however, due to its toxicity to the human body, nicotine

\footnotetext{
- Received September 14, 2020

Revised December 5, 2020

Accepted December 9, 2020

- Corresponding author: Yong-Dae Kim

Department of Otorhinolaryngology-Head and Neck Surgery, Yeungnam

University College of Medicine, 170 Hyeonchung-ro, Nam-gu,

Daegu 42415, Korea

Tel: +82-53-620-3781, Fax: +82-53-628-7884

E-mail: ydkim@med.yu.ac.kr
}

insecticides have not been commercially available in the United States since 2014 and have been banned in the EU since 2009 [1]. It is well known that the common side effects of nicotine exposure include cerebrovascular and cardiac problems, as well as psychiatric disorders. Moreover, nicotine is known to act as a tumor promoter. Although nicotine exerts only minor effects on the respiratory system, recent studies have suggested that chronic exposure to nicotine can change the rheological properties of human airway mucus function and cause respiratory stimulation, such as tachypnea. Nicotine can also induce bronchial muscle contraction and respiratory paralysis at toxic doses [2].

Tobacco detoxification allows tobacco manufacturers to develop and sell cigarettes categorized as "light," "mild," "lowtar," or "low nicotine" to reflect the reduced tobacco content.

Copyright @ 2021 by Korean Society of Otorhinolaryngology-Head and Neck Surgery.

This is an open-access article distributed under the terms of the Creative Commons Attribution Non-Commercial License (https://creativecommons.org/licenses/by-nc/4.0)

which permits unrestricted non-commercial use, distribution, and reproduction in any medium, provided the original work is properly cited. 
Nicotine content has been widely used as an indicator of tobacco toxicity, and many smokers actually perceive these "lowernicotine" products to be less harmful [3]. Recently, the development of electronic cigarettes (e-cigarettes), which do not leave a lingering smell after smoking, has resulted in a rapid increase in e-cigarette consumption worldwide. Various types of e-cigarettes are available, ranging from liquid nicotine to which specific flavorings can be added, to products that already contain nicotine and other chemicals, to heat-not-burn cigarettes. Furthermore, manufacturers claim that e-cigarettes are less harmful than traditional cigarettes, and that the smell after smoking is also significantly reduced. It is estimated that many traditional smokers use e-cigarettes, and reports suggest that they may help with smoking cessation or at least limit conventional cigarette use.

However, the impact of e-cigarette vapor on the lungs compared with traditional cigarette smoke is not fully understood. The toxicity of e-cigarette vapor with or without nicotine has also been poorly studied. Therefore, in the present study, we focused on the effects of e-cigarette vapor with or without nicotine in the liquid on mucin production by human airway epithelial cells.

\section{MATERIALS AND METHODS}

This study was approved by the Institutional Review Board for human studies at the Yeungnam University Medical Center (IRB No. 2018-04-004) and written informed consent was obtained from all 10 patients.

\section{Materials}

Nicotine was purchased from Sigma (N0267-100MG; St. Louis, MO, USA). A commercially available e-cigarettes device, eGo ONE CT (length $131.2 \mathrm{~mm} \times 19 \mathrm{~mm}$; battery capacity $2200 \mathrm{mAh}$; atomizer capacity $2.5 \mathrm{~mL} ; 1.8 \mathrm{~mm}$ air hole; resistance $1.0 \mathrm{ohm}$; providing $15 \mathrm{~W}$ power) was used for vapor production, and was purchased from Shenzhen Joyetec (ShenZhen, China). Propylene glycol (PG) and vegetable glycerin (VG) were obtained from Alfa Aesar (Karlsruhe, Germany), with a purity of $99.5 \%$.

NCI-H292 airway epithelial cells were obtained from the American Type Culture Collection (Manassas, VA, USA). EpiLife

\section{H I G G H L I I G H T S}

- Electronic cigarettes (e-cigarettes) stimulated MUC5AC expression, but not MUC5B expression regardless of nicotine content.

- The mitogen-activated protein kinase (MAPK) and MAPK pathways induce MUC5AC expression.

- E-cigarette vapors have potentially harmful effects regardless of nicotine content. growth medium and human keratinocyte growth supplements were obtained from Cascade Biologics (Portland, OR, USA). Airway epithelial cell growth medium was purchased from PromoCell (Heidelberg, Germany). For primary culture, nasal mucosa tissues were obtained from normal inferior turbinate samples from 10 patients undergoing augmentation rhinoplasty who had no personal or family history of allergy and who had displayed negative results on skin-prick tests to 20 common airborne allergens and on multiple simultaneous allergen tests.

\section{Extract production}

E-cigarettes liquid was prepared without nicotine and with $24 \mathrm{mg} / \mathrm{mL}$ nicotine [4,5]. Both liquids contained 50\% PG and $50 \%$ VG. e-cigarette vapor was extracted by simulating use. The e-cigarettes device was connected to a $50-\mathrm{mL}$ syringe through a sealed tube. In the absence of a standardized vaping profile for e-cigarettes, inhalation simulation lasted 4 seconds, one puff every 60 seconds between inhalations [6]. E-cigarette vapor media from $0.5 \mathrm{~mL}$ and $1 \mathrm{~mL}$ e-liquid (with or without nicotine) in cartomizer cartridges was bubbled through $20 \mathrm{~mL}$ serum-free RPMI media either 20 or 40 times [4]. E-cigarette vapor was generated by manual activation of the heating coil with constant air compression with a 20 seconds puff duration. $\mathrm{pH}$ was adjusted to 7.4 , and the solution was filtered through a $0.22 \mu \mathrm{m}$ syringe filter [7].

\section{Treatment and exposure}

NCI-H292 cells were cultured in RPMI 1640 supplemented with $2 \mathrm{mM}$ L-glutamine, $100 \mathrm{U} / \mathrm{mL}$ penicillin, $100 \mu \mathrm{g} / \mathrm{mL}$ streptomycin, and $10 \%$ fetal bovine serum at $37^{\circ} \mathrm{C}$ in $5 \% \mathrm{CO}_{2}$ with humidified air. When cells reached $80 \%-90 \%$ confluence, they were serum-starved for 24 hours then rinsed with phosphatebuffered saline (PBS) and exposed to e-cigarettes vapor with or without nicotine [8]. E-cigarette vapor was extracted using 0 , 20 , and 40 times per $50 \mathrm{~mL}$ syringe and mixed with $20 \mathrm{~mL}$ serum-free RPMI 1640 media. These values are based on the fact that e-cigarettes users took $13 \pm 2$ puffs at 5 minutes and $43 \pm 8$ puffs at 20 minutes.

Nasal mucosal tissues were washed with PBS and immersed in dispase for 90 minutes. After the tissue was scraped off the surface of the nasal mucosa with a scalpel, tissues were added to $1 \%$ PBS and filtered through a mesh. The suspension was then centrifuged at 3,000 rpm for 5 minutes. The cells were then seeded in the wells of a 24 -well plate at $2.5 \times 10^{5}$ cells/well and incubated in EpiLife medium and human keratinocyte growth supplement. When cultures were confluent, cells were exposed to e-cigarette vapor in the same ways as the NCI-H292 cells were exposed. Procreation and activity of NCI-H292 cells and nasal epithelial cells were quantified using an EZ-Cytox Cell Viability Assay Kit (Daeil Lab, Seoul, Korea), and morphological changes were observed by microscopy. We have performed at least three times for each test. 


\section{Reverse transcription-polymerase chain reaction}

A GeneAmp RNA PCR core kit (Thermo Fisher Scientific, Foster City, CA, USA) was used to analyze mRNA expression. Primer sequences and polymerase chain reaction (PCR) conditions were as previously described [9]. Glyceraldehyde-3-phosphate dehydrogenase (GAPDH) was used as a positive control.

\section{Real-time PCR}

Real-time (RT)-PCR was practiced using iQ SYBR Green Supermix (Bio-Rad, Hercules, CA, USA). Quantitative PCR was performed using a Bio-Rad CFX96 RT-PCR system C1000 Thermal Cycler with 40 cycles of $95^{\circ} \mathrm{C}$ for 10 seconds primer-specific annealing temperatures for 5 seconds, and $62^{\circ} \mathrm{C}$ for 10 seconds. GAPDH was used as an internal control. Amplification specificity was measured using melting curves, by the manufacturer's instructions.

\section{Enzyme-linked immunosorbent assay}

NCI-H292 cells and human nasal epithelial cells were treated with e-cigarette vapor with or without nicotine, as described above. After further incubation for 24 hours, MUC5AC protein levels were determined using enzyme-linked immunosorbent assay (ELISA). Lysates sample from epithelial cells were incubated overnight at $4^{\circ} \mathrm{C}$, then plates were blocked with $2 \%$ bovine serum albumin (BSA) and incubated with the primary antibody in PBS containing $0.05 \%$ Tween 20 for 1 hour. Wells were washed and treated with the secondary antibody for 1 hour. Color was developed using 3,3',5,5'-tetramethylbenzidine peroxidase solution, and the reaction was terminated with $2 \mathrm{~N}$ $\mathrm{H}_{2} \mathrm{SO}_{4}$. Optical density measurements were obtained using an EL800 ELISA reader (BioTek Instruments, Winooski, VT, USA) at $450 \mathrm{~nm}$. Results are expressed as fold increase above baseline controls.

\section{Western blot analysis}

NCI-H292 cells were seeded onto a 6-well plate and treated with the indicated concentrations of e-cigarette vapor with or without nicotine. Western blot analysis of extracellular signalregulated kinase (ERK)1/2, p38 mitogen-activated protein kinase (MAPK), and nuclear factor kappa-light-chain-enhancer of activated B cells (NF- $\mathrm{kB}$ ) phosphorylation was performed as previously described [9].

\section{Cell transfection with siRNAs}

NCI-H292 cells were seeded onto a 6-well plate. When the cells reached $80 \%-90 \%$ confluence, OPTI-MEN I reduced serum medium was added. ERK1 MAPK small interfering RNA (siRNA) and Lipofectamine 2000 (Life technologies, Carlsbad, CA, USA) were cultivated together in Opti-MEM I reduced serum medium (Life technologies) for 20 minutes to form an ERK1 MAPK siRNA-Lipofectamine complex, which was then supplemented to each well at a final siRNA concentration of $100 \mathrm{nM}$.
Transfected cells were incubated for 48 hours at $37^{\circ} \mathrm{C}$ in a $\mathrm{CO}_{2}$ incubator then exposed to the indicated concentrations of e-cigarette vapor with or without nicotine. Transfection efficacy was $>90 \%$. The same procedure was performed for control, MAPK (ERK1/2 and p38), and NF-kB.

\section{Ex vivo tissue culture and immunofluorescence}

To confirm the signaling pathway involved in e-cigarette vaporinduced MUC5AC expression in human nasal inferior turbinate tissue, we performed immunofluorescence (IF) staining in $e x$ vivo culture. Before proceeding with the culture, the tissues were washed three times with PBS. After 8 hours of e-cigarette vapor (with or without nicotine $24 \mathrm{~g} / \mathrm{mL}$ ) treatment, tissue fragments were fixed in $4 \%$ paraformaldehyde, after which tissues were washed with PBS. Fixed tissues were embedded with optimal cutting temperature compound for 1 hour at $-80^{\circ} \mathrm{C}$. IF analyses were performed on $6-\mu \mathrm{m}$ thick sections and blocked in $1 \%$ BSA in phosphate buffered saline with $0.1 \%$ Tween 20 for 1 hour at room temperature. The slides were incubated with a rabbit anti-MUC5AC antibody (ab-198294; Abcam, Cambridge, UK; 1:100 dilution). Subsequently, they were incubated with an Alexa 488-labelled goat anti-rabbit immunoglobulin G secondary antibody (green fluorescence). Nuclei were stained with 4',6-diamidino-2-phenylindole (DAPI; blue fluorescence, Abcam). The cells were visualized by fluorescence microscopy using Nikon software (Ti-S, 733551; Nikon,Tokyo, Japan).

\section{Statistical analysis}

Statistical analysis was performed using SPSS ver. 12.0 (SPSS Inc., Chicago, IL, USA). Data are represented as mean \pm standard deviation. Comparisons were accomplished using an unpaired $t$-test or Kruskal-Wallis test followed by a Mann-Whitney test. For all tests, $P$-values $<0.05$ were reflected statistically significant.

\section{RESULTS}

\section{Measurement of cytotoxicity}

The cytotoxicity of e-cigarette vapor on NCI-H292 cells and human nasal epithelial cells was assessed using water-soluble tetrazolium salt-1. NCI-H292 cells and human nasal epithelial cells were treated with e-cigarette vapor (with or without nicotine $24 \mathrm{mg} / \mathrm{mL}$ ) for 24 hours. E-cigarette vapor did not display visible cell killing activity at up to $2 \mathrm{~mL}$ of e-liquid vapor (Fig. 1).

\section{E-cigarette vapor (with or without nicotine) stimulated MUC5AC expression in human $\mathrm{NCl}-\mathrm{H} 292$ cells}

To evaluate the effects of e-cigarette vapor on MUC5AC and MUC5B expression, NCI-H292 cells were incubated with different doses of e-cigarette vapor for 8 hours. RT-PCR analysis showed that e-cigarette vapor (with or without nicotine $24 \mathrm{mg} / \mathrm{mL}$ ) stim- 

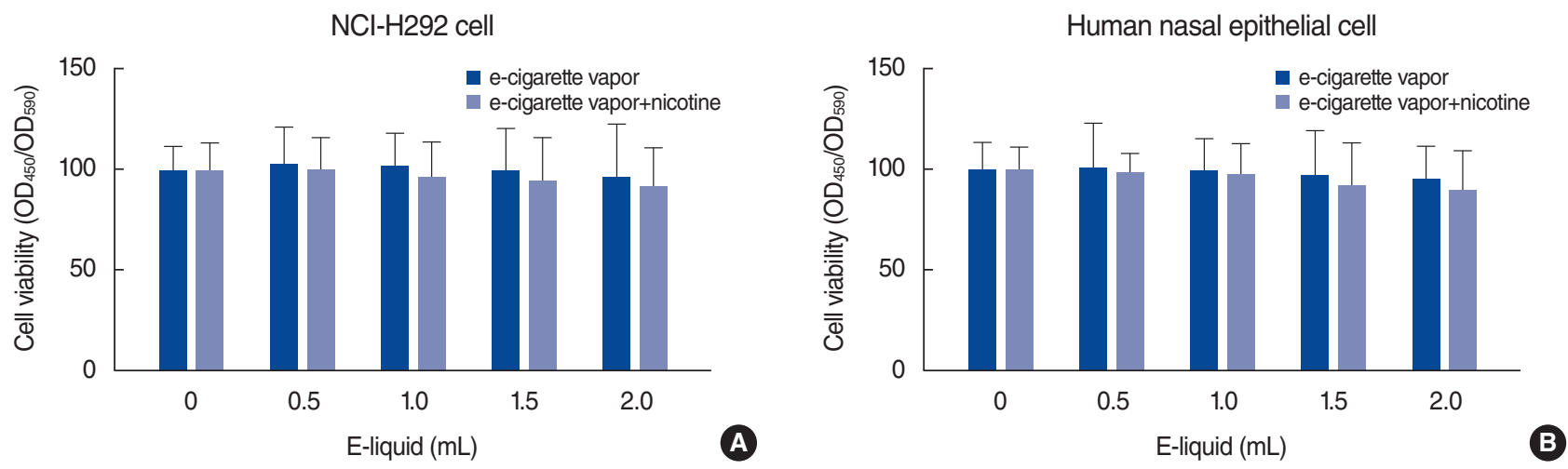

Fig. 1. Effects of e-cigarette vapor (with or without nicotine) on NCl-H292 cells and human nasal epithelial cells. (A) Result of cytotoxicity testing of NCl-H292 cells with water-soluble tetrazolium salt-1 (WST-1) treatment, showing that e-cigarette vapor did not display visible cell killing activity with up to $2 \mathrm{~mL}$ of e-liquid vapor when incubated for 24 hours. (B) Result of cytotoxicity testing of human nasal epithelial cells with WST-1 treatment, showing that e-cigarette vapor did not display visible cell killing activity with up to $2 \mathrm{~mL}$ of e-liquid vapor when incubated for 24 hours. e-cigarette, electronic cigarettes.
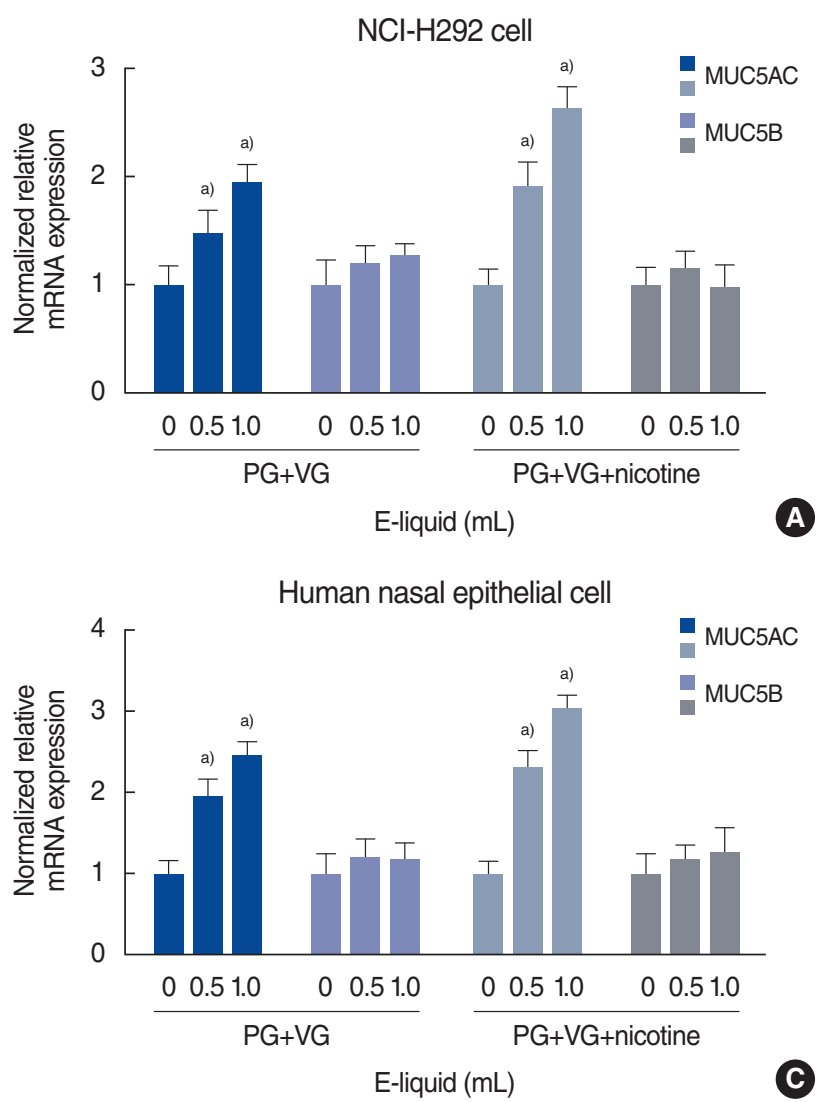
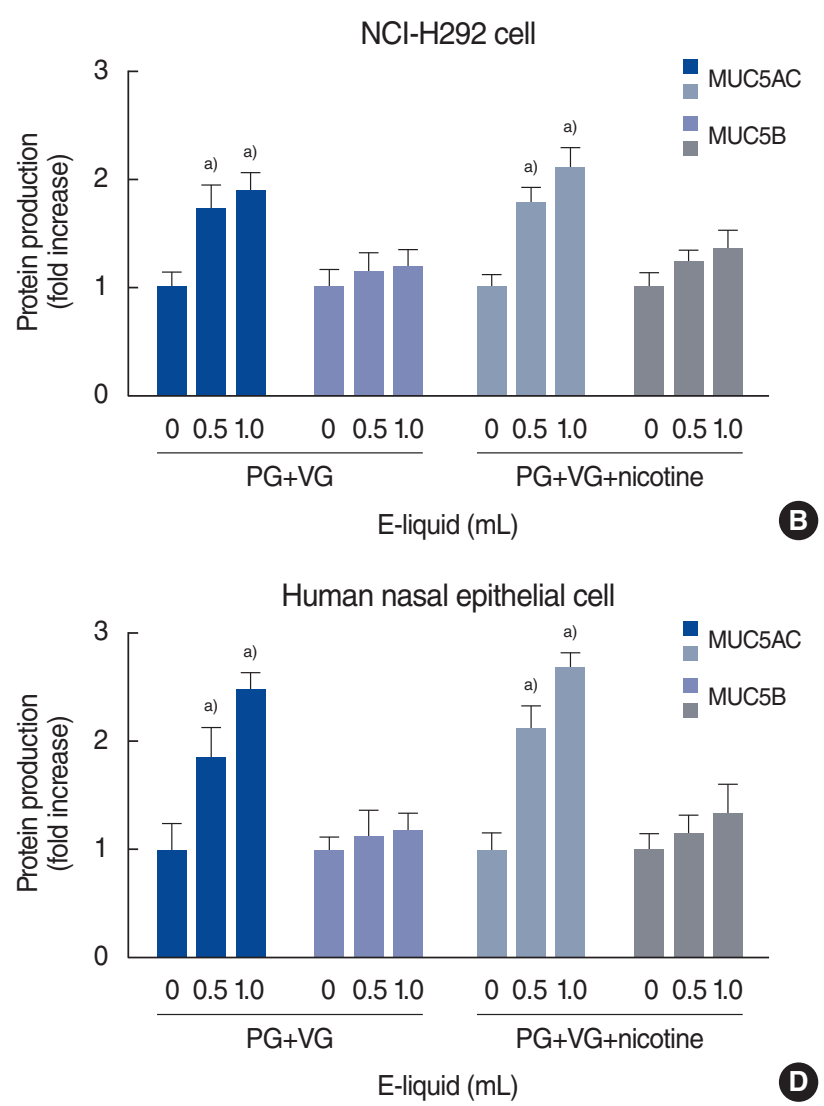

Fig. 2. Effects of e-cigarette vapor (with or without nicotine) on airway mucin expression in NCl-H292 cells and human nasal epithelial cells. (A) Real-time polymerase chain reaction (RT-PCR) results showing that e-cigarette vapor (with or without nicotine $24 \mathrm{mg} / \mathrm{mL}$ ) meaningfully stimulated MUC5AC, but not MUC5B, mRNA expression in NCl-H292 cells. (B) Enzyme-linked immunosorbent assay (ELISA) results showing that e-cigarette vapor (with or without nicotine $24 \mathrm{mg} / \mathrm{mL}$ ) significantly stimulated MUC5AC, but not MUC5B, protein levels in NCl-H292 cells. (C) RT-PCR results showing that e-cigarette vapor (with or without nicotine $24 \mathrm{mg} / \mathrm{mL}$ ) meaningfully stimulated MUC5AC, but not MUC5B, mRNA expression in human nasal epithelial cells. (D) ELISA results showing that e-cigarette vapor (with or without nicotine $24 \mathrm{mg} / \mathrm{mL}$ ) significantly increased MUC5AC, but not MUC5B, protein levels in human nasal epithelial cells. Bars represent mean \pm standard deviation of three indepen-

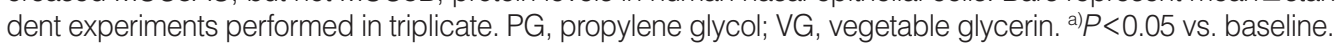


ulated MUC5AC expression, but not MUC5B expression, in the cells (Fig. 2A). The ELISA results also indicated that e-cigarette vapor (with or without nicotine $24 \mathrm{mg} / \mathrm{mL}$ ) significantly increased MUC5AC protein production (Fig. 2B).

\section{E-cigarette vapor}

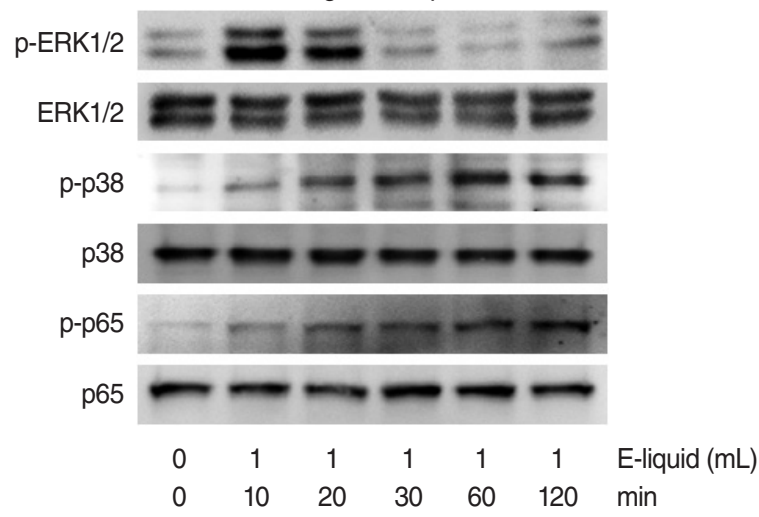

E-cigarette vapor

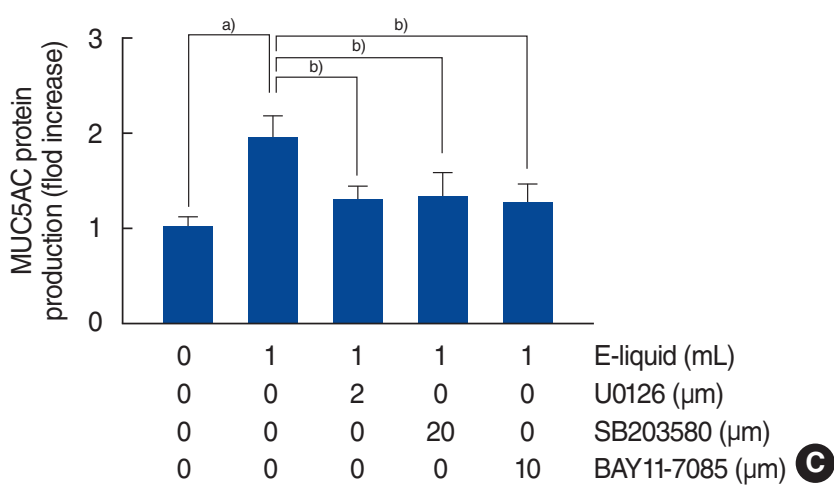

E-cigarette vapor

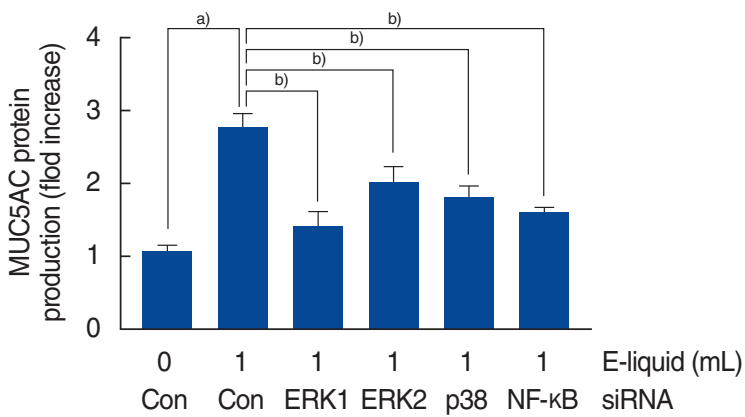

The increase in MUC5AC expression in human NCl-H292 cells due to e-cigarette vapor (with or without nicotine) involved ERK1/2, p38, and NF-KB activation

To investigate the relationship between MAPK (ERK1/2 and p38) and NF- $\mathrm{B}$ signaling and e-cigarette vapor-induced MUC5AC

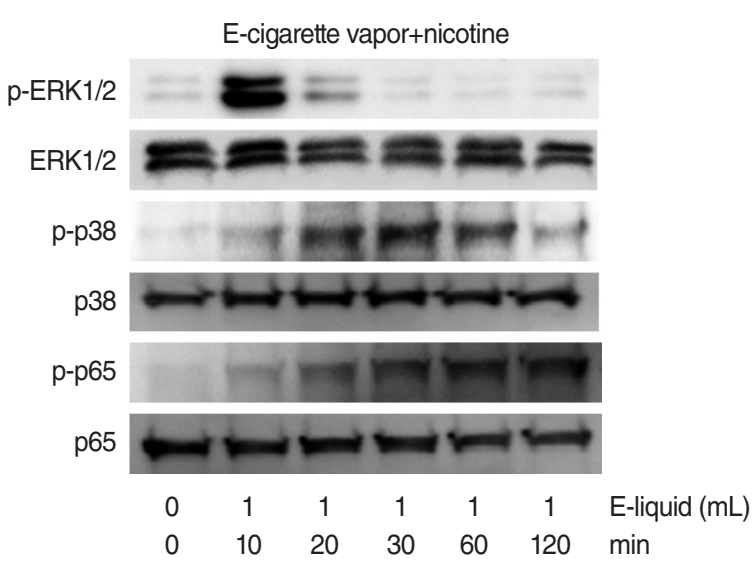

B
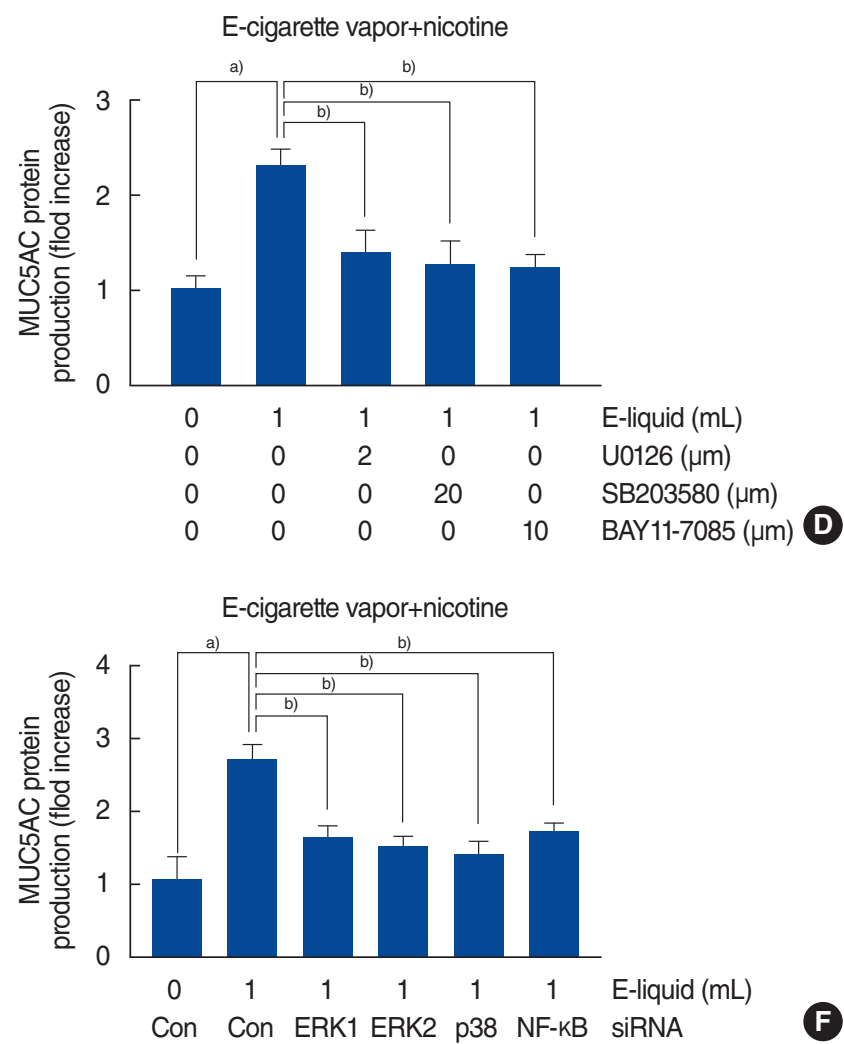

Fig. 3. MUC5AC overexpression by e-cigarette vapor (with or without nicotine) in human airway NCl-H292 epithelial cells involves ERK1/2, p38, and NF-kB stimulation. (A, B) Western blot analysis showing that e-cigarette vapor with or without nicotine induced phosphorylation of ERK1/2, p38, and NF-kB. (C, D) Enzyme-linked immunosorbent assay (ELISA) results showing that U0126 (an ERK1/2 inhibitor), SB203580 (a p38 inhibitor), and BAY 11-7085 (an NF-kB inhibitor) treatment significantly attenuated the effect of e-cigarette vapor on MUC5AC protein levels. (E, F) ELISA results showing that knockdown of ERK1, ERK2, p38, and NF-kB by siRNA significantly blocked the effect of e-cigarette vapor on MUC5AC protein levels. Images are illustrative of three separate experiments performed in triplicate. Bars show mean \pm standard deviation of three independent experiments performed in triplicate. e-cigarette, electronic cigarettes; ERK, extracellular signal-regulated kinase; pERK, phosphorylated ERK; p-p38, phosphorylated p38; p-p65, phosphorylated p65; Con, control; NF-kB, nuclear factor kappa-light-chain-enhancer of activated B cells; siRNA, small interfering RNA. a) $P<0.05$ vs. baseline. ${ }^{\text {b) }} P<0.05$ vs. e-cigarette vapor alone. 
expression, NCI-H292 airway epithelial cells were treated with e-cigarette vapor for 8 hours andWestern blots were used to analyze MAPK (ERK1/2 and p38) and NF- $\mathrm{kB}$ phosphorylation. The results showed that e-cigarette vapor (with or without nicotine $24 \mathrm{mg} / \mathrm{mL}$ ) activated ERK1/2 and p38 (Fig. 3A and B). Then, to determine whether the ERK1/2, p38, and NF-kB signaling pathways are involved in e-cigarette-induced MUC5AC expression, the cells were pretreated with MAPK inhibitors. Treatment with U0126 (a specific ERK1/2 inhibitor), SB203580 (a specific p38 inhibitor), or BAY 11-7085 (a specific NF-кB inhibitor) significantly attenuated the MUC5AC protein production induced by e-cigarettes (with or without nicotine $24 \mathrm{mg} / \mathrm{mL}$ ) (Fig. 3C and D). To further support these findings, cells were transfected with ERK1, ERK2, p38 MAPK, or NF- $\kappa$ B siRNA constructs, then exposed to e-cigarette vapor (with or without nicotine $24 \mathrm{mg} / \mathrm{mL}$ ) for 8 hours. Knockdown of ERK1, ERK2, and p38 significantly blocked the MUC5AC mRNA protein production induced by ecigarettes (with or without nicotine $24 \mathrm{mg} / \mathrm{mL}$ ) (Fig. 3E and F).

E-cigarette vapor (with or without nicotine) increased MUC5AC expression in human primary nasal epithelial cells

E-cigarette vapor (with or without nicotine $24 \mathrm{mg} / \mathrm{mL}$ ) did not

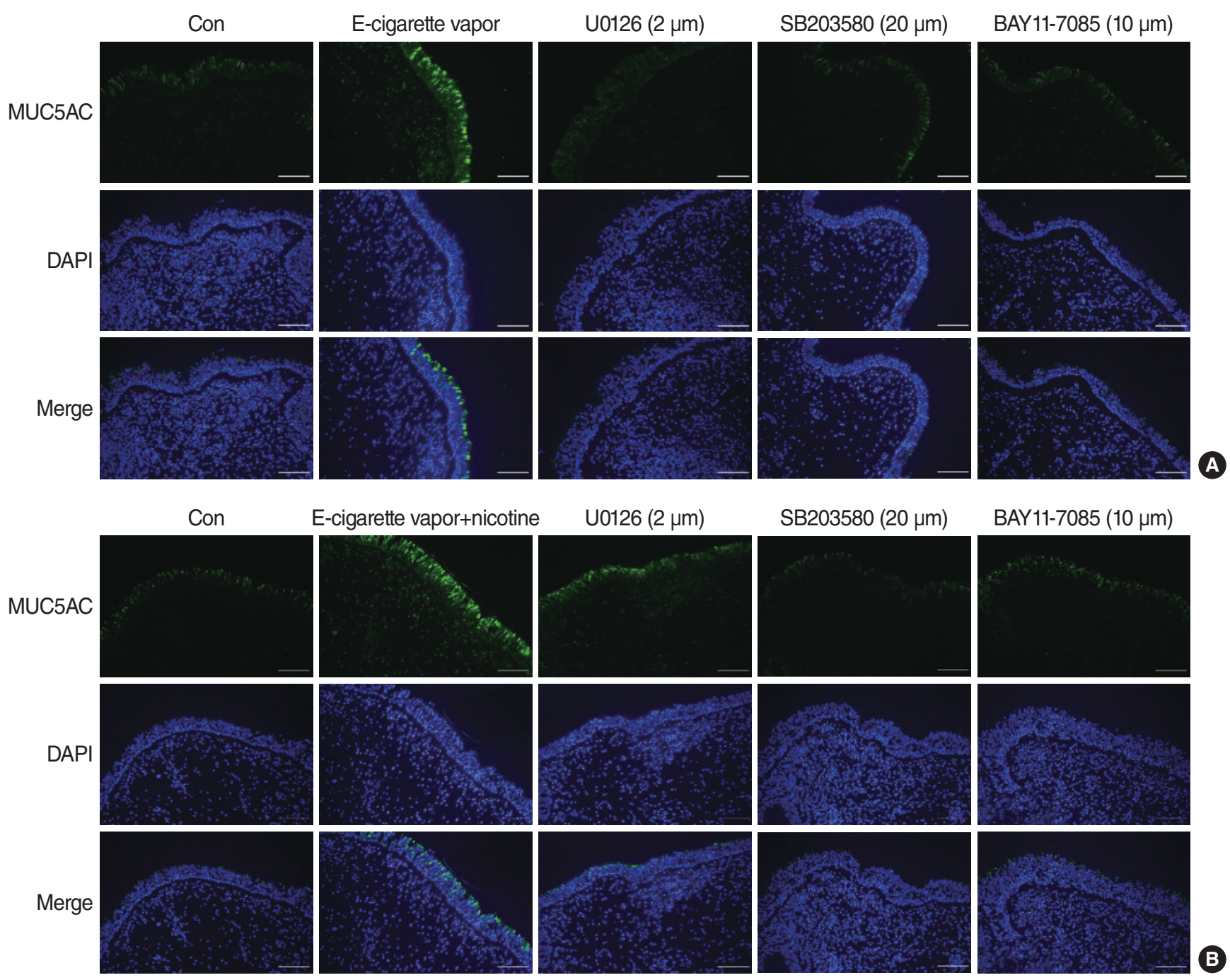

Fig. 4. E-cigarette vapor (with or without nicotine)-induced MUC5AC expression in human nasal epithelial cells is associated with ERK1/2, p38, and NF-kB activation. (A) Immunofluorescence results showing that e-cigarette vapor without nicotine significantly induced MUC5AC expression, and this was significantly inhibited by pretreatment with U0126 (an ERK1/2 inhibitor), SB203580 (a p38 inhibitor), or BAY 11-7085 (an NF$\mathrm{KB}$ inhibitor). E-cigarette vapor without nicotine also significantly increased MUC5AC expression in the cytoplasm. Each bar shows the mean \pm standard deviation of three independent experiments. (B) Immunofluorescence results showing that e-cigarette vapor with nicotine significantly increased MUC5AC expression, and this was significantly inhibited by pre-treatment with U0126 (an ERK1/2 inhibitor), SB203580 (a p38 inhibitor), or BAY 11-7085 (an NF-kB inhibitor). Furthermore, e-cigarette vapor with nicotine significantly increased MUC5AC expression in the cytoplasm. Con, control; e-cigarette, electronic cigarettes; DAPI, 4',6-diamidino-2-phenylindole; ERK, extracellular signal-regulated kinase; NF$\mathrm{kB}$, nuclear factor kappa-light-chain-enhancer of activated B cells. 
affect the viability of human nasal epithelial cells when used at concentrations of up to $2 \mathrm{~mL}$ of e-liquid vapor (Fig. 1B). E-cigarette vapor (with or without nicotine $24 \mathrm{mg} / \mathrm{mL}$ ) induced mRNA expression and protein production of MUC5AC, but not MUC5B, in human nasal epithelial cells (Fig. 2C and D).

\section{E-cigarette vapor (with or without nicotine)-stimulated MUC5AC expression in cultures of human nasal inferior turbinate tissue involved ERK1/2, p38, and NF-KB signaling}

To confirm the signaling pathways involved in e-cigarette-induced MUC5AC protein production in human nasal inferior turbinate tissue, we performed IF analysis of ex vivo cultures. Treatment with U0126, SB2035800, or BAY 11-7085 (specific pathway inhibitors) significantly attenuated the effects of e-cigarette vapor (with or without nicotine $24 \mathrm{mg} / \mathrm{mL}$ ) on MUC5AC protein production in the epithelium (Fig. 4).

\section{DISCUSSION}

Airway mucus is important for maintaining homeostasis of the respiratory system, where it functions to control epithelial viscosity and tracheobronchial mucociliary clearance. An abnormal increase or decrease in mucus impairs the mucociliary transport system and can lead to lung diseases such as asthma, chronic bronchitis, and chronic obstructive pulmonary disease. The viscoelastic properties of airway mucus depend on two major secretory mucin glycoproteins, MUC5AC and MUC5B.

Recent studies have reported the effects of e-cigarette vapor on airway mucin production. Reidel et al. [10] reported that the MUC5AC concentration in the sputum of e-cigarette smokers was significantly higher than that of nonsmokers, while MUC5B levels were not affected by e-cigarette use. Ghosh et al. [11] reported that vapor from e-cigarettes without nicotine resulted in a 3- to 4-fold increase in MUC5AC levels, and that no further increase was observed following exposure to e-cigarette vapor with nicotine. In addition, they reported that MUC5B levels were unchanged after exposure of bronchial epithelial cells to ecigarette vapor. In the present study, we found that e-cigarette vapor with or without nicotine meaningfully amplified MUC5AC expression in airway epithelial cells, while MUC5B was not affected. We therefore focused on MUC5AC expression to assess the effect of e-cigarette vapor on human airway epithelial cells. and showed that e-cigarette vapor with or without nicotine increased activity of the MAPK and NF- $\mathrm{BB}$ signaling pathways. Experiments were performed using representative cell lines of the upper and lower airway epithelial cells to confirm similarities and differences in the effect of e-cigarette vapor with or without nicotine on MUC5AC and MUC5B expression, which is one of the factors suspected to exacerbate upper and lower inflammatory airway diseases.

E-cigarettes are devices that can vaporize a nicotine solution, which can be combined with various liquid flavorings. The main components of e-cigarettes' liquid phase are PG, VG, flavorings, and nicotine. PG and VG act as mediators of nicotine and flavor delivery and as humectants for the smoker's mouth, throat, and lungs, but are less well-studied than nicotine. E-cigarette users can combine liquids with various PG:VG content or can purchase commercialized liquid products according to their preferences. PG is a synthetic compound that is odorless, colorless, viscous, and mildly sweet. It is used in a variety of applications, from food processing to polymer synthesis, and is also used as an ingredient in e-cigarettes to control throat stimulation. Glycerin is commonly used in daily life, especially in medicine, food, and beauty products, and is also used as a humectant and coloring material. It is used as an ingredient in e-cigarettes, to control the amount of smoke produced during use. PG and VG are reported to have very low toxicity when administered orally and are recognized as safe additives by the Food and Drug Administration. However, while the gastrointestinal system can easily digest these compounds, inhalation of smoke through e-cigarettes may have toxic effects. Alexander et al. [12] reported that PG aerosols caused an increase in goblet cell number and mucin production in the nose. Renne et al. [13] reported evidence suggesting that VG aerosol exposure may result in mild squamous metaplasia of the epithelial lining of the epiglottis. In addition, Lechasseur et al. [14] reported that the major solvents used in e-cigarettes, including PG and VG but not nicotine or flavorings, could alter the expression of genes that control the molecular clock, and showed that the regulatory genes $h s p a 1 a$ and $h s p a 1 b$ were altered in the lungs [14]. In the current study, we showed that vapor from PG and VG liquid meaningfully amplified MUC5AC expression in airway epithelial cells compared to controls.

Nicotine is a major toxic component of tobacco. In the respiratory system, nicotine induces MUC5AC expression, which increases mucin production, and alters the innate immune response. Inhaled nicotine vapor is associated with chronic airway disease and lung remodeling. Lerner et al. [15] reported that acute exposure to e-cigarette aerosols stimulated mitochondrial sensitivity, disrupted mitochondrial energy, and induced nuclear DNA fragmentation. Garcia-Arcos et al. [16] reported that chronic exposure to inhaled nicotine from e-cigarettes promoted the development of obstructive airway disease by increasing cytokine expression, airway hyper-reactivity, and lung tissue destruction. In an in vivo study, nicotine-containing vapor was found to cause significantly more endothelial permeability than nicotine-free vapor, as well as rapidly increasing lung injury and oxidative stress [17]. However, in the current study, we showed that nicotine-containing e-cigarette vapor did not significantly induce MUC5AC expression, compared with vapor not containing nicotine.

Several studies have highlighted the potential positive health effects of e-cigarette smoking. For example, Margham et al. [18] reported that e-cigarette vapor was less complex than cigarette smoke and contained significantly lower levels of toxins. Gonie- 
wicz et al. [19] showed that while e-cigarettes did contain toxic compounds such as formaldehyde, acetaldehyde, acrolein, toluene, $\mathrm{N}$-nitrosonornicotine, and 4-(methylnitrosamino)-1-(3pyridyl)-1-butanone (NNK), the levels were 9- to 450-fold high$\mathrm{er}$ in smoke from traditional cigarettes than in e-cigarette vapor. However, it is very difficult to obtain conclusive results on the toxicological and immunological effects of e-cigarette vapor. However, recent studies have reported that e-cigarette smoke causes various biological alterations and exacerbates pathology. Hiemstra and Bals [20] showed that e-cigarette vapor induced adverse effects, ranging from reduced viability, an increase in the production of inflammatory mediators, and a reduction in antimicrobial defenses to pro-carcinogenic changes in both cell culture and in vivo models. More specifically, exposure to excessive e-cigarette vapor induces the production of proinflammatory cytokines such as interleukin (IL)-8, IL-10, interferon-gamma, and vascular endothelial growth factor by human alveolar epithelial cells, and also stimulates inflammatory responses, including MAPK, Janus tyrosine kinase/signal transducer and activator of transcription, and NF- $\mathrm{kB}$ signaling in human alveolar epithelial cells [21]. Chronic exposure to e-cigarette vapor with or without nicotine has been shown to induce the proinflammatory effect of endothelial barrier disruption via oxidative stress, p38 MAPK activation, increased intracellular ceramides, and myosin light chain phosphorylation [17]. In the current study, we showed that e-cigarette vapor, with or without nicotine, increased the activity of the MAPK and NF- $\mathrm{kB}$ signaling pathways, and experiments were performed using representative cell lines of the upper and lower airway epithelial cells to confirm similarities and differences in the effect of e-cigarette vapor with or without nicotine on MUC5AC and MUC5B expression, which is one of the factors suspected to exacerbate upper and lower inflammatory airway diseases.

We showed that e-cigarette vapor with or without nicotine induced MUC5AC expression in human airway epithelial cells via activation of MAPK and NF-kB signaling. Vapor from e-liquid containing only PG and VG also significantly increased MUC5AC expression, and this was not further increased by vapor from e-liquid containing PG, VG, and nicotine. Therefore, vapor from only PG and VG may be associated with an increase in MUC5AC expression and may have a toxic effect.

A limitation of this study is that we used only one popular ecigarette device, and it is possible that our findings may not apply to other brands. There are no standard definitions of e-cigarettes devices and liquids; different manufacturers make various types of e-cigarettes that incorporate a range of different ingredients. Moreover, there is no generally established general toxic level of e-cigarette vapor containing PG and VG. Therefore, we determined the toxic level through WST testing. To summarize, our data show that even brief exposure to e-cigarette vapor can alter mucin production and stimulate mechanisms that may contribute to lung disease.

\section{CONFLICT OF INTEREST}

No potential conflict of interest relevant to this article was reported.

\section{ACKNOWLEDGMENTS}

This research was supported by the Basic Science Research Program through the National Research Foundation of Korea (NRF) funded by the Ministry of Science, ICT \& Future Planning (NRF2018R1A2B6007756 to YDK).

This work was supported by a 2017 Yeungnam University Research Grant (No. 217A380113).

\section{ORCID}

Si-Youn Song https://orcid.org/0000-0001-6642-8841

Hyung Gyun Na https://orcid.org/0000-0003-4017-4796

So Young Kwak https://orcid.org/0000-0001-9386-8121

Yoon Seok Choi https://orcid.org/0000-0002-0616-7122

Chang Hoon Bae https://orcid.org/0000-0002-0835-4060

Yong-Dae Kim https://orcid.org/0000-0003-0501-966X

\section{AUTHOR CONTRIBUTIONS}

Conceptualization: HGN, SYS, YDK. Data curation: SYS, YSC, SYK. Formal analysis: CHB, YSC. Funding acquisition: SYS, YDK. Methodology: HGN, CHB. Project administration: SYS, YDK. Visualization: SYK, CHB. Writing-original draft: HGN, YDK.Writing-review \& editing: SYS, YDK.

\section{REFERENCES}

1. Thornton SL, Oller L, Sawyer T. Fatal intravenous injection of electronic nicotine delivery system refilling solution. J MedToxicol. 2014 Jun;10(2):202-4.

2. Chen EY, Sun A, Chen CS, Mintz AJ, ChinWC. Nicotine alters mucin rheological properties. Am J Physiol Lung Cell Mol Physiol. 2014 Jul;307(2):L149-57.

3. Kozlowski LT, Dreschel NA, Stellman SD, Wilkenfeld J, Weiss EB, Goldberg ME. An extremely compensatible cigarette by design: documentary evidence on industry awareness and reactions to the Barclay filter design cheating the tar testing system. Tob Control. 2005 Feb;14(1):64-70.

4. Farsalinos KE, Romagna G, Tsiapras D, Kyrzopoulos S, Voudris V. Evaluation of electronic cigarette use (vaping) topography and estimation of liquid consumption: implications for research protocol standards definition and for public health authorities' regulation. Int J Environ Res Public Health. 2013 Jun;10(6):2500-14.

5. Wu Q, Jiang D, Minor M, Chu HW. Electronic cigarette liquid increases inflammation and virus infection in primary human airway epi- 
thelial cells. PLoS One. 2014 Sep;9(9):e108342.

6. Farsalinos KE, Romagna G, Allifranchini E, Ripamonti E, Bocchietto $\mathrm{E}$, Todeschi S, et al. Comparison of the cytotoxic potential of cigarette smoke and electronic cigarette vapour extract on cultured myocardial cells. Int J Environ Res Public Health. 2013 Oct;10(10): 5146-62.

7. Martey CA, Pollock SJ, Turner CK, O’Reilly KM, Baglole CJ, Phipps $\mathrm{RP}$, et al. Cigarette smoke induces cyclooxygenase- 2 and microsomal prostaglandin E2 synthase in human lung fibroblasts: implications for lung inflammation and cancer. Am J Physiol Lung Cell Mol Physiol. 2004 Nov;287(5):L981-91.

8. Farsalinos KE, Romagna G, Tsiapras D, Kyrzopoulos S, Voudris V. Evaluating nicotine levels selection and patterns of electronic cigarette use in a group of "vapers" who had achieved complete substitution of smoking. Subst Abuse. 2013 Sep;7:139-46.

9. Kwak S, Kim YD, Na HG, Bae CH, Song SY, Choi YS. Resistin upregulates MUC5AC/B mucin gene expression in human airway epithelial cells. Biochem Biophys Res Commun. 2018 May;499(3):655-61.

10. Reidel B, Radicioni G, Clapp PW, Ford AA, Abdelwahab S, Rebuli $\mathrm{ME}$, et al. E-cigarette use causes a unique innate immune response in the lung, involving increased neutrophilic activation and altered mucin secretion. Am J Respir Crit Care Med. 2018 Feb;197(4):492501.

11. Ghosh A, Coakley RC, Mascenik T, Rowell TR, Davis ES, Rogers K, et al. Chronic e-cigarette exposure alters the human bronchial epithelial proteome. Am J Respir Crit Care Med. 2018 Jul;198(1):67-76.

12. Alexander DJ, Collins CJ, Coombs DW, Gilkison IS, Hardy CJ, Healey G, et al. Association of Inhalation Toxicologists (AIT) working party recommendation for standard delivered dose calculation and expression in non-clinical aerosol inhalation toxicology studies with pharmaceuticals. Inhal Toxicol. 2008 Oct;20(13):1179-89.
13. Renne RA, Wehner AP, Greenspan BJ, Deford HS, Ragan HA, Westerberg RB, et al. 2-Week and 13-week inhalation studies of aerosolized glycerol in rats. Inhal Toxicol. 1992;4(2):95-111.

14. Lechasseur A, Jubinville E, Routhier J, Berube JC, Hamel-Auger M, Talbot M, et al. Exposure to electronic cigarette vapors affects pulmonary and systemic expression of circadian molecular clock genes. Physiol Rep. 2017 Oct;5(19):e13440.

15. Lerner CA, Rutagarama P,AhmadT, Sundar IK, Elder A, Rahman I. Electronic cigarette aerosols and copper nanoparticles induce mitochondrial stress and promote DNA fragmentation in lung fibroblasts. Biochem Biophys Res Commun. 2016 Sep;477(4):620-5.

16. Garcia-Arcos I, Geraghty P, Baumlin N, Campos M, Dabo AJ, Jundi $\mathrm{B}$, et al. Chronic electronic cigarette exposure in mice induces features of COPD in a nicotine-dependent manner. Thorax. 2016 Dec; 71(12):1119-29.

17. Schweitzer KS, Chen SX, Law S, Van Demark M, Poirier C, Justice MJ, et al. Endothelial disruptive proinflammatory effects of nicotine and e-cigarette vapor exposures. Am J Physiol Lung Cell Mol Physiol. 2015 Jul;309(2):L175-87.

18. Margham J, McAdam K, Forster M, Liu C, Wright C, Mariner D, et al. Chemical composition of aerosol from an e-cigarette: a quantitative comparison with cigarette smoke. Chem Res Toxicol. 2016 Oct; 29(10):1662-78.

19. Goniewicz ML, Knysak J, Gawron M, Kosmider L, Sobczak A, Kurek $\mathrm{J}$, et al. Levels of selected carcinogens and toxicants in vapour from electronic cigarettes. Tob Control. 2014 Mar;23(2):133-9.

20. Hiemstra PS, Bals R. Basic science of electronic cigarettes: assessment in cell culture and in vivo models. Respir Res. 2016 Oct;17(1):127.

21. Kaur G, Pinkston R, Mclemore B, Dorsey WC, Batra S. Immunological and toxicological risk assessment of e-cigarettes. Eur Respir Rev. 2018 Feb;27(147):170119. 